\title{
Individual differences in eating and drinking in the rat'
}

\section{JAMES ALLISON; Indiana L'niversity, Bloomingtom, Ind. 47401}

In a sample of four rats, highly reliable individual differences were found on the variables which determint' drinking rate and eating rate: the interval between successive licks $(p<.001)$, and the interval between successive contacts with food $(\mu<.005)$, within bursts of drinking and eating, respectively. Interials within bursts of eating were longer $(p<.01)$ and more variable than intervals within bursts of drinking. Individual differences accounted for .23 of the variance in the case of drinking, and .03 of the variance in the case of eating.

Whether rats show reliable individual differences in rates of drinking and eating has become a matter of some concern in the theoretical analysis of reinforcement. Premack (1965) has proposed that the principles which underlie the reinforcing properties of drinking and eating may be derivable from S's "operating characteristics"-rates of drinking and eating measured under conditions in which $S$ has free access to food and water. He suggested as a working assumption that where rate of drinking is concerned, rats show no individual differences in their operating characteristics: when rats drink. drinking rate is invariant at about seven licks/sec. On the contrary, there is some reason to doubt the alleged invariance of lick rate. Mean lick rate varies very little from one study to the next (Davis \& Keehn. 1959: Schaeffer \& Premack. 1961: Stellar \& Hill, 19521. and the ranges generally appear rather small to the naked eye. but there seems to be only one reported attempt to test the statistical significance of individual differences in lick rate. In a paper not cited by Premack. Keehn \& Arnold (1960) reported lick rates ranging from 6.64 to $7.52, \mathrm{sec}$ in a sample of eight rats: the difference between the two extreme $S$ s was significant at $p<.001$ However. they did not report their statistical test procedure. nor their rationale for discarding all but the two extreme $S s$ in testing the null hypothesis.

Considerably less is known about eating rate. Premack (1965) concluded from his own. and apparently the only such. investigation that rats eat $45 \mathrm{mg}$ Noyes pellets at about one pellet $/ 6.5$ sec. However. he reported few methodological details. and no test of the statistical significance of individual differences.

The purpose of this experiment was to determine whether rats differ reliably in rates of eating and drinking. Previous investigations have used graphic recording devices with resolutions on the order of . $02 \mathrm{sec}$ at best. It was thought that if reliable individual differences exist at this level of behavior. they might be rathar small. Measures were therefore obtained with a resolution of $.001 \mathrm{sec}$. This resolution was made possible by a decision to measure directly the variable which determines drinking rate. the time between successive licks within a burst of drinking. The same approach was lised in dealing with eating rate. since a preliminary study of the topography of eating indicated that rats eat mash in bursts that can be defined pretty clearly. Successive contacts with the food are either separated by a relatively short interval during which $\mathrm{S}$ makes gross chewing movements and stays close to the source of food. or by a much longer interval during which $S$ may leave the food. and stops chewing before returning for the next contact. Total contacts and total amount eaten were highly correlated (rho $=.90 . \mathrm{N}=7 . \mathrm{p}<.01)$.

Subjects

The Ss were four male albino rats, approximately 150 days

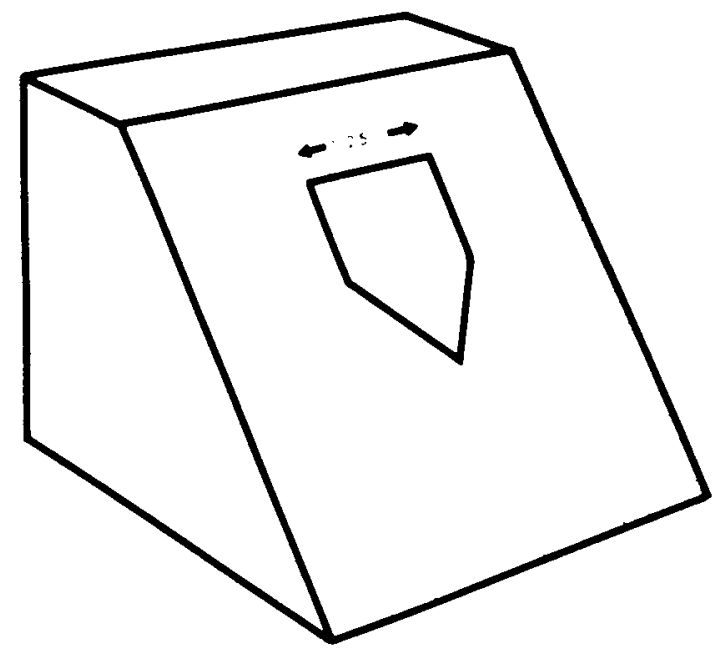

Fig. 1. Food bin.

old, purchased from Windsor Biology Gardens (Bloomington. Indiana).

Apparatus

The $15 \times 8 \times 8 \mathrm{in}$. test enclosure consisted of four plywood walls painted midgray, a clear Plexiglas ceiling. and a grid floor made of 19 stainless steel tubes with a diameter of $3 / 8$ in. spaced about $3 / 4$ in. on center. Centered laterally on one end wall. a glass drinking tube with a $3-\mathrm{mm}$ aperture extended $1 / 2$ in. into the enclosure, $1 \mathrm{in}$. off the floor. A clear Plexiglas food bin was centered laterally on the other end wall. $1 / 2$ in. off the floor. The food bin was designed in such a way. that $S$ did not paw the food, and could not eat except by thrusting the head into the bin (see Fig. 1). A metal plate on the floor of the bin was covered with a 1/4in. layer of damp mash, sufficiently conductive to operate a drinkometer reliably (four parts of finely ground Noyes pellets to one part of water. by weight).

A lick at the drinking tube activated a drinkometer supplied by BRS Electronics. Automatic control circuitry was programmed in such a way that when $E$ enabled the recording system by pressing a button. the next lick started a .001-sec electronic clock (Hunter Digital Counter-Timer. Model 1520). The following lick stopped the clock. and disabled the recording system. The measure thus obtained was the interlick interval (onset to onset). referred to hereafter as the drink interval. A second identical system was used for eating. When E enabled the system. the next contact with the food in the bin started. and the following contact stopped a .001-sec clock. measuring the interfood-contact interval (referred to hereafter as the eat intervall.

Table 1

Drink and Eat Intervals in Sec for Individual Ss

\begin{tabular}{lcccc}
\hline & \multicolumn{4}{c}{ Subject } \\
\cline { 2 - 5 } Statistic & 1 & 2 & 3 & + \\
& & Drink & & \\
\hline Mean & .256 & .130 & .178 & .142 \\
SD & .128 & .032 & .104 & .001 \\
& & Eat & & \\
\hline Mean & .702 & .840 & .834 & .485 \\
SD & .786 & .911 & .724 & $.55+$
\end{tabular}




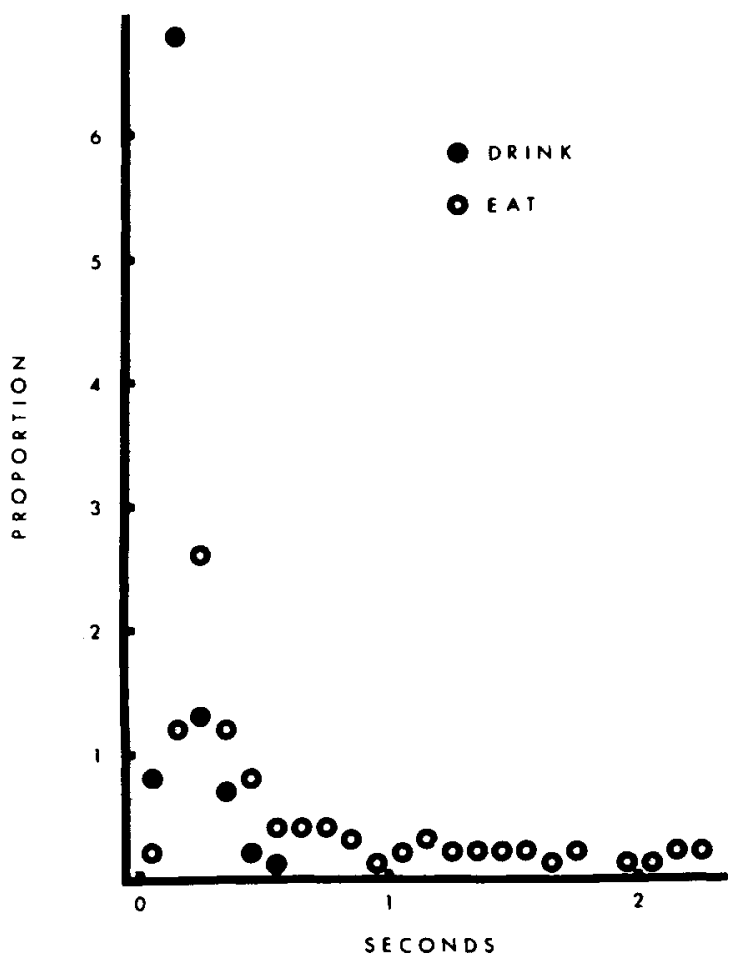

Fig. 2. Distributions of drink intervals and eat intervals (Class Intervals $=.099 \mathrm{sec}$ ) .

The test enclosure was illuminated by four diffused $30-\mathrm{W}$ fluorescent lights about $5 \mathrm{ft}$ overhead, and white masking noise was present at all times.

Procedure

During the 10-day period prior to the first day of testing the Ss were fed and watered $1 \mathrm{~h}$ daily, and were accustomed to eating and drinking in the test enclosure from the food bin and the drinking tube. Drink intervals and eat intervals were measured beginning at $23 \mathrm{~h}$ of food and water deprivation, in one or two test sessions. No session was allowed to exceed $1 \mathrm{~h}$, and the testing of a given $S$ was discontinued when 100 drink and 100 eat intervals had been obtained from that $S$. The total of 800 observations included only those intervals which clearly occurred within a burst of drinking or eating; one E observed S by an overhead mirror and signaled to a second $E$, who enabled the recording system and recorded the clock reading. Results and Discussion

Mean drink intervals ranged from .130 to .256 , with a grand mean of $.176 \mathrm{sec}$. Individual means and standard deviations appear in the upper part of Table 1. In order to test the significance of individual differences using a random effects model, it was necessary to determine whether successive intervals were independent. An autocorrelation of the form $r_{n, n}+1$, where $n=(-1,2, \ldots, 98,99)$ was calculated for each $\mathrm{S}$. The autocorrelations ranged from -.13 to .28 , mean $\mathrm{r}=.02$, indicating that successive intervals could be considered independent. The one-way analysis of variance indicated highly reliable individual differences in drink interval
( $F=40.36, \mathrm{df}=3 / 396, \mathrm{p}<.001)$. Mean squares between and within Ss were .321 and .008 , respectively.

Mean eat intervals ranged from .485 to .840 , with a grand mean of $.715 \mathrm{sec}$. Individual means and standard deviations appear in the lower part of Table 1. Autocorrelations ranged from -.09 to .14 , mean $r=.02$. Individual differences in eat interval were highly reliable $(F=4.87, d f=3 / 396, p<.005)$. Mean squares between and within Ss were 2.773 and .570 , respectively.

As Table 1 shows, eat intervals were consistently longer $(t=6.34, \mathrm{df}=3, \mathrm{p}<.01)$ and more variable than drink intervals. The two relative frequency distributions appear in Fig. 2.

It appears that the significant differences among Ss could have been detected with clocks having less resolution than the clocks used here. Additional analyses of intervals rounded to the nearest .1 sec revealed significant individual differences in both drinking $(\mathrm{p}<.001)$ and eating $(\mathrm{p}<.005)$.

Contrary to Premack's supposition, rats engaged in eating or drinking show reliable individual differences in their operating characteristics, but it is difficult to assess the practical importance of these differences. Granted that they are highly reliable, are they large or small relative to differences produced by other sources of variance? The numerical magnitude of a variation is not very meaningful in this regard, since it can be made arbitrarily large or small by an appropriate transformation of the measures. Less arbitrary is the proportion of variance accountable to a particular independent variable (Hays, 1963). This statistic provides a meaningful scale of the relative importance of various sources of variance, provided it is a population estimate. The latter proviso means that $E$ must have selected the values of each independent variable at random, from a well defined population of values (e.g., various magnitudes of food reward selected at random from a clearly specified set of possible magnitudes)-a rare occurrence in psychological research, except in investigations of individual differences. The foregoing considerations indicate the difficulty of comparing subject variables with other independent variables in terms of their relative importance as sources of behavioral variance. It is easier to determine whether individual differences account for more behavioral variance on one dependent variable than another. In the present case individual differences accounted for a considerably greater proportion of variance in drinking than in eating, the proportions being .23 and .03 respectively.

\section{REFERENCES}

DAVIS, J. D \& KEEHN, J. D. Magnitude of reinforcement and consummatory behavior. Science, 1959, 130, 269-270.

HAYS, W. L. Statistics for psychologists. New York: Holt, Rinehart, \& Winston, 1963.

KEEHN, J. D., \& ARNOLD, E. M. M. Licking rates of albino rats. Science, 1960, 132, 739-741.

PREMACK, D. Reinforcement theory. In D. Levine (Ed.), Nebraska sympasium on mativation, 1965. Lincoln: University of Nebraska Press, 1965. Pp. 123-180.

SCHAEFFER, R. W. \& PREMACK, D. Licking rates in infant albino rats. Science, $1961,132,1980-1981$.

STELLAR, E., \& HIL, J. H. The rat's rate of drinking as a function of water deprivation. Journal of Comparative \& Physiological Psychology, 1952, 45, 96-102.

\section{NOTE}

1. Supported by United States Public Health Service Grant MH 11470-03. My thanks to C. Fruechtenicht, D. Kennerty, W. Powers, and S. Rhodes for their assistance. 